\title{
Dose-response relationship between genetically proxied average blood glucose levels and incident coronary heart disease in individuals without diabetes mellitus
}

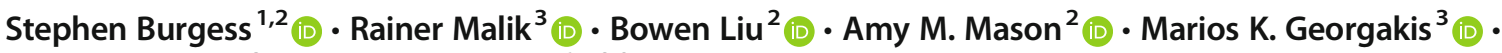 \\ Martin Dichgans $^{3,4,5}$ (D) Dipender Gill ${ }^{6,7,8,9}$ (iD
}

Received: 25 September 2020 / Accepted: 19 November 2020 / Published online: 26 January 2021

(C) The Author(s) 2021

\begin{abstract}
Aims/hypothesis Our aim was to investigate the relationship between average blood glucose levels and incident CHD in individuals without diabetes mellitus.

Methods To investigate average blood glucose levels, we studied $\mathrm{HbA}_{1 \mathrm{c}}$ as predicted by 40 variants previously shown to be associated with both type 2 diabetes and $\mathrm{HbA}_{1 \mathrm{c}}$. Linear and non-linear Mendelian randomisation analyses were performed to investigate associations with incident CHD risk in 324,830 European ancestry individuals from the UK Biobank without diabetes mellitus.

Results Every one $\mathrm{mmol} / \mathrm{mol}$ increase in genetically proxied $\mathrm{HbA}_{1 \mathrm{c}}$ was associated with an $11 \%$ higher $\mathrm{CHD}$ risk (HR $1.11,95 \%$ CI 1.05, 1.18). The dose-response curve increased at all levels of $\mathrm{HbA}_{1 \mathrm{c}}$, and there was no evidence favouring a non-linear relationship over a linear one.

Conclusions/interpretations In individuals without diabetes mellitus, lowering average blood glucose levels may reduce CHD risk in a dose-dependent way.
\end{abstract}

Keywords Average blood glucose levels $\cdot$ CHD $\cdot$ Mendelian randomisation

\section{Introduction}

CHD is a leading cause of mortality, accounting for approximately 9 million deaths globally in 2015 alone [1]. Epidemiological studies have supported an association between average blood glucose levels and CHD risk, even in individuals without diabetes mellitus [2]. However, it is difficult to infer causal effects from observational studies because of the possibility that any identified associations may be

Dipender Gill

dgill@sgul.ac.uk

1 Medical Research Council Biostatistics Unit, Cambridge Institute of Public Health, Cambridge, UK

2 Department of Public Health and Primary Care, University of Cambridge, Cambridge, UK

3 Institute for Stroke and Dementia Research, University Hospital of Ludwig-Maximilians-University, Munich, Germany

4 Munich Cluster for Systems Neurology, Munich, Germany

5 German Centre for Neurodegenerative Diseases, Munich, Germany attributable to confounding. Mendelian randomisation can overcome these limitations by using genetic variants as instrumental variables to infer the effect of modifying an exposure such as average blood glucose levels on an outcome such as CHD. The aim of this study was to perform linear and nonlinear Mendelian randomisation analyses to investigate the shape of the causal relationship between average blood glucose levels (measured by $\mathrm{HbA}_{1 \mathrm{c}}$ ) and $\mathrm{CHD}$ risk in individuals without diabetes mellitus. $\mathrm{HbA}_{1 \mathrm{c}}$ was considered

6 Clinical Pharmacology and Therapeutics Section, Institute of Medical and Biomedical Education and Institute for Infection and Immunity, St George's, University of London, London, UK

7 Clinical Pharmacology Group, Pharmacy and Medicines Directorate, St George's University Hospitals NHS Foundation Trust, London, UK

8 Novo Nordisk Research Centre Oxford, Old Road Campus, Oxford, UK

9 Department of Epidemiology and Biostatistics, School of Public Health, Imperial College London, London, UK 


\section{Research in context}

\section{What is already known about this subject?}

- While epidemiological studies have supported an association between average blood glucose levels and CHD risk in individuals without diabetes mellitus, it is difficult to infer causal effects because of possible confounding

- Mendelian randomisation can overcome this limitation using genetic variants as instrumental variables to infer the effect of modifying an exposure such as average blood glucose levels on an outcome such as CHD

\section{What is the key question?}

- What is the shape of the causal relationship between average blood glucose levels, as measured by $\mathrm{HbA}_{1 c}$ and $\mathrm{CHD}$ risk in individuals without diabetes mellitus?

\section{What are the new findings?}

- We found genetic evidence supporting an effect of higher average blood glucose levels on increasing CHD risk in individuals without diabetes mellitus

- There was no evidence favouring a non-linear relationship between $\mathrm{HbA}_{1 \mathrm{c}}$ and $\mathrm{CHD}$ risk over a linear one

\section{How might this impact on clinical practice in the foreseeable future?}

- This work will raise awareness that lowering average blood glucose levels may, in certain contexts, reduce cardiovascular risk in individuals without diabetes mellitus

preferable to blood glucose levels because it represents the average blood glucose level over approximately 120 days, so is less susceptible to variation related to the time of recording.

\section{Methods}

Analyses were performed in unrelated participants of European ancestry from the UK Biobank, a populationbased cohort study of middle-aged UK residents. Individuals having possible diabetes mellitus (defined based on selfreporting, hospital episode statistics and medications) or baseline $\mathrm{HbA}_{1 \mathrm{c}}>47.5 \mathrm{mmol} / \mathrm{mol}(6.5 \%)$ were excluded. $\mathrm{HbA}_{1 \mathrm{c}}$ was measured in packed red blood cells using the Bio-Rad Variant II Turbo analyser. International Classification of Diseases 9th Revision (ICD-9) codes 410-414, and ICD-10 codes I20-I25 were used to identify incident CHD cases. Full details are provided in the electronic supplementary material (ESM).

Candidate instrumental variables We selected 40 uncorrelated $\left(r^{2}<0.001\right)$ single-nucleotide polymorphisms as instrumental variables for average blood glucose levels based on their association with type 2 diabetes $\left(p<5 \times 10^{-8}\right)$ in a genome-wide association study of 228,499 cases and 1,178,783 controls (79\% European ancestry) that included UK Biobank participants [3], and their association with $\mathrm{HbA}_{1 \mathrm{c}}(p<0.001$ and concordant direction of association) in an independent study of 100,880 European ancestry participants (no overlap with the UK Biobank) that were free of diabetes mellitus (as defined by physician diagnosis, medications, or fasting glucose $\geq 7 \mathrm{mmol} / \mathrm{l}$ ) [4] (ESM Table 1). We created a weighted allele score for each participant by multiplying each type 2 diabetes risk-increasing allele dosage with the variant's association with $\mathrm{HbA}_{1 \mathrm{c}}$, and summing across all 40 variants. Selecting variants associated with type 2 diabetes and weighting by their association with $\mathrm{HbA}_{1 \mathrm{c}}$ helps ensure that the weighted allele score is reflective of average blood glucose levels, rather than solely type 2 diabetes risk or $\mathrm{HbA}_{1 \mathrm{c}}$.

Statistical analysis Mendelian randomisation analyses were performed to investigate the association between genetically proxied average blood glucose levels (measured as $\mathrm{HbA}_{1 \mathrm{c}}$ ) and incident CHD. Analyses were performed by modelling a linear relationship between genetically proxied average blood glucose levels and incident CHD ('linear Mendelian randomisation') [5], and also using the fractional polynomial method to test for a non-linear relationship between genetically proxied average blood glucose levels and incident CHD ('non-linear Mendelian randomisation') [6]. We further assessed associations of the allele score with lipid fractions and other glycaemic traits, and performed multivariable nonlinear Mendelian randomisation for traits associated with the allele score that may represent alternative causal risk factors. As a further sensitivity analysis, we also performed Mendelian randomisation analysis that excluded variants associated with the alternative causal risk factor at $p<0.01$. Full details are provided in the ESM. All statistical analysis was performed using R (version 3.6.2) and only publicly available data from studies that had obtained relevant ethical approval and participant consent were used. 


\section{Results}

Baseline characteristics for the 324,830 participants included in the analyses are provided in ESM Table 2. There were 6006 incident CHD events. The allele score explained $1.8 \%$ of the variance in $\mathrm{HbA}_{1 \mathrm{c}}$, corresponding to an F-statistic of 144.5 and a low risk of substantial weak instrument bias. For UK Biobank participants, associations of the variants incorporated in the allele score with $\mathrm{HbA}_{1 \mathrm{c}}$ were generally of greater magnitude in men compared with women (ESM Fig. 1).

Linear Mendelian randomisation Linear Mendelian randomisation analyses identified a positive association between higher genetically proxied average blood glucose levels and incident $\mathrm{CHD}$ risk when considering men and women together (ESM Fig. 2). For a one $\mathrm{mmol} / \mathrm{mol}$ increase in $\mathrm{HbA}_{1 \mathrm{c}}$, the $\mathrm{HR}$ for

a

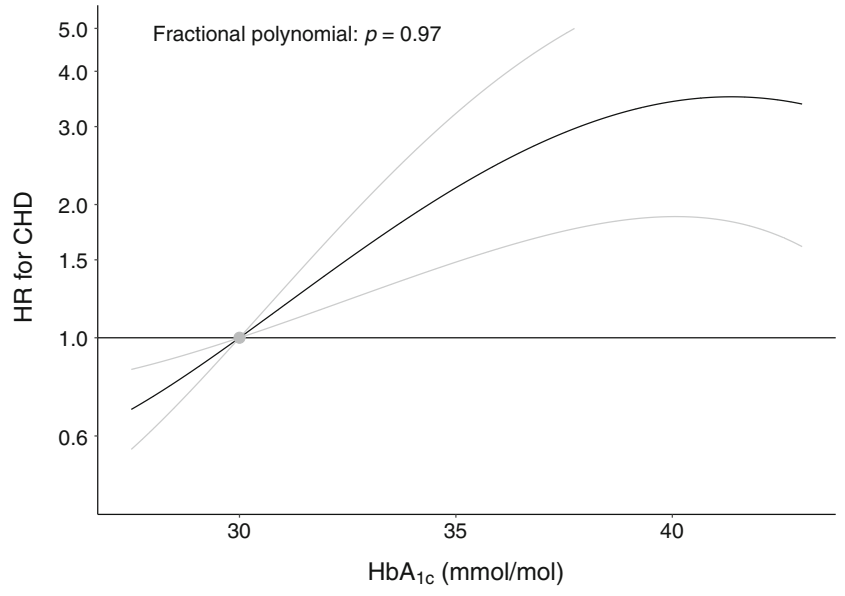

incident CHD was 1.11 (95\% CI 1.05, 1.18; $\left.p=2 \times 10^{-4}\right)$. In sex-stratified analyses, the association was stronger in men (HR $1.12,95 \%$ CI 1.05, 1.19; $p=4 \times 10^{-4}$ ) than in women (HR 1.08, 95\% CI 0.96, 1.20; $p=0.20$ ) (ESM Table 3). Similar point estimates were obtained in sensitivity analyses using alternative Mendelian randomisation methods (ESM Table 4).

Non-linear Mendelian randomisation In non-linear Mendelian randomisation, we observed no statistical evidence favouring a non-linear relationship between genetically proxied $\mathrm{HbA}_{1 \mathrm{c}}$ and incident CHD over a linear one in any of the analyses (Fig. 1). Subgroup analyses presenting Mendelian randomisation estimates in quintiles of the population based on $\mathrm{HbA}_{1 \mathrm{c}}$ levels (corrected for genetic predisposition) are presented in ESM Table 3.

b

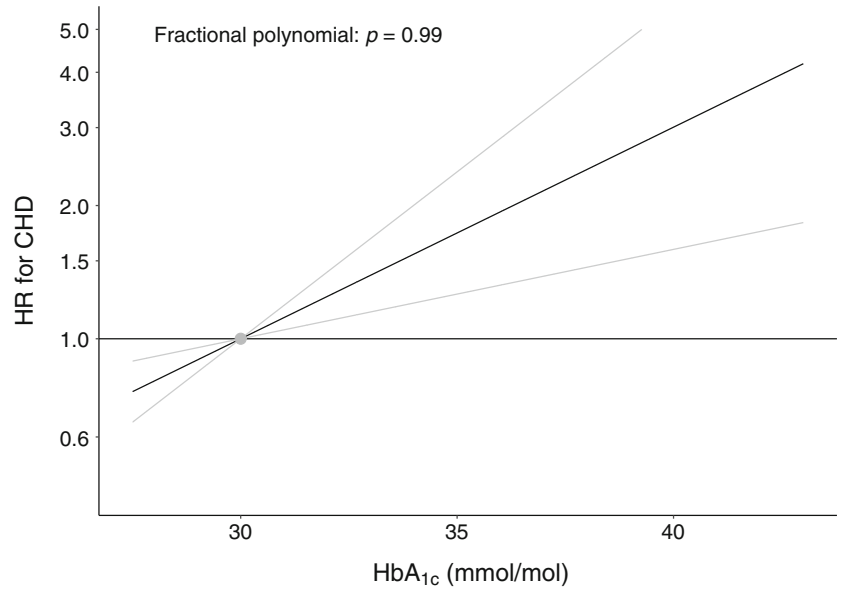

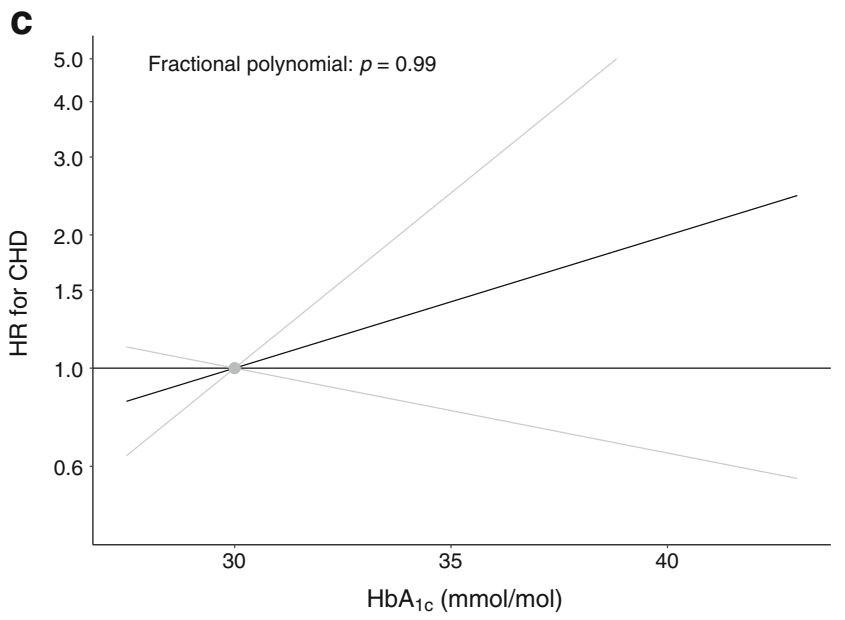

Fig. 1 Non-linear Mendelian randomisation investigating the relationship between genetically proxied average blood glucose levels (measured by $\mathrm{HbA}_{1 \mathrm{c}}$ ) and risk of incident $\mathrm{CHD}$ in individuals without diabetes mellitus: (a) men and women combined; (b) men only; and (c) women only. The $x$-axis depicts $\mathrm{HbA}_{1 \mathrm{c}}$ levels in $\mathrm{mmol} / \mathrm{mol}$. The $y$-axis depicts the hazard ratio for coronary heart disease (HR for CHD) with respect to the reference, plotted on a log scale. Reference is set to an $\mathrm{HbA}_{1 \mathrm{c}}$ of $30 \mathrm{mmol} /$ mol (4.9\%). The grey lines represent the $95 \%$ CIs. The fractional polynomial test is a goodness-of-fit test that assesses whether any improvement of fit when using a non-linear function to model the association, compared with a linear function, is greater than would be expected due to chance (a significant $p$ value indicates that a non-linear model is preferred to a linear model) 
We assessed genetic associations of the allele score and its individual variants with other glycaemic traits and lipid fractions (ESM Table 5 and ESM Fig. 3). The allele score was associated with 2-h glucose $(p<0.001)$ and fasting glucose $(p<0.001)$, but not fasting insulin $(p=0.22)$. The allele score was also associated with LDL-cholesterol (LDL-cholesterol, $p=0.03$ ), but not HDLcholesterol $(p=0.86)$ or triacylglycerols $(p=0.99)$. Although multivariable non-linear Mendelian randomisation adjusting for genetically proxied LDL-cholesterol showed some attenuation in the coefficient for genetically proxied $\mathrm{HbA}_{1 \mathrm{c}}$, an association persisted (HR 1.07, 95\% CI 1.01, 1.14; $p=0.018$ ), and the bestfitting fractional polynomial was the linear model (ESM Fig. 4). Similar results were also obtained after excluding the five variants that associated with LDL-cholesterol at $p<0.01$ (rs1260326, rs10184004, rs11708067, rs505922 and rs174541): HR 1.10 (95\% CI 1.03, 1.17; $p=0.003$ ) (ESM Fig. 5).

\section{Discussion}

In this Mendelian randomisation study, we found genetic evidence supporting an effect of higher average blood glucose levels on increasing CHD risk in individuals without diabetes mellitus. We did not find evidence favouring a non-linear relationship between $\mathrm{HbA}_{1 \mathrm{c}}$ and $\mathrm{CHD}$ risk over a linear one. Our findings provide evidence that lowering average blood glucose levels in individuals without diabetes mellitus can reduce cardiovascular risk in a dose-dependent way.

While pre-diabetes is known to increase cardiovascular risk [7], our work goes further to support a continuous effect of average blood glucose levels that are within the 'physiologically normal' $\mathrm{HbA}_{1 \mathrm{c}}$ range. Our findings build on existing epidemiological research supporting an association between average blood glucose levels and CVD risk in individuals without diabetes mellitus [2]. A previous Mendelian randomisation study similarly showed a positive association between genetically proxied $\mathrm{HbA}_{1 \mathrm{c}}$ and $\mathrm{CHD}$ risk, although with wider CIs than in our current work, likely because that work used fewer genetic variants as instrumental variables [8]. Clinical trials have found that intensive lowering of $\mathrm{HbA}_{1 \mathrm{c}}$ levels in high-risk patients with type 2 diabetes does not confer a beneficial effect on cardiovascular risk $[9,10]$. This discrepancy may be related to the particular pharmacological treatments used to lower $\mathrm{HbA}_{1 \mathrm{c}}$ levels, including adverse effects such as weight gain, hypoglycaemia and rapid fluctuations in glucose levels $[9,10]$. Pharmacological agents for blood glucose lowering that are not associated with weight gain or hypoglycaemia are available [11]. Clinical trials are warranted to explore whether particular blood glucose lowering strategies can be used to reduce cardiovascular risk in patients without diabetes mellitus.

A strength of our study is that the employed Mendelian randomisation approach is robust to confounding from environmental factors. The weights for the variants used in Mendelian randomisation analysis were derived from their associations with $\mathrm{HbA}_{1 \mathrm{c}}$ in a dataset that did not include the UK Biobank [4]. Furthermore, analysis was restricted to UK Biobank participants free of type 2 diabetes, thus avoiding any influence of winner's curse bias. We further performed sensitivity analyses that accounted for potential genetic confounding through LDL$\mathrm{C}$, which also identified associations of genetically proxied $\mathrm{HbA}_{1 \mathrm{c}}$ levels with incident CHD risk. However, our study also has limitations. The analyses were restricted to UK Biobank participants of European ancestry, and may not apply to other populations. Furthermore, the genetic variants employed as instrumental variables only explained $1.8 \%$ of the variance in $\mathrm{HbA}_{1 \mathrm{c}}$, thus limiting the statistical power of the analyses and the precision of the results. A reason why the Mendelian randomisation estimates were less precise in women compared with men may be that the genetic variants had weaker associations with $\mathrm{HbA}_{1 \mathrm{c}}$ in women. Finally, the genetic variants employed as instruments in this analysis proxy lifelong average blood glucose control, and therefore cannot be used to inform on the quantitative effects of discrete clinical interventions that lower blood glucose levels in the short term.

In summary, this Mendelian randomisation analysis provides genetic evidence supporting an effect of average blood glucose levels on CHD risk in individuals without diabetes mellitus. Further work is required to investigate whether strategies that lower blood glucose levels can reduce cardiovascular risk in individuals without diabetes mellitus.

Supplementary Information The online version of this article (https://doi. org/10.1007/s00125-020-05377-0) contains peer-reviewed but unedited supplementary material.

Data availability This research was performed using UK Biobank data (application 29202), which is available on request (https://www. ukbiobank.ac.uk/register-apply). The genetic variants used as instrumental variables are detailed in ESM Table 1.

Funding SB is supported by a Sir Henry Dale Fellowship jointly funded by the Wellcome Trust and the Royal Society (204623/Z/16/Z). MKG is funded by a scholarship from the Onassis Foundation. MD acknowledges funding from the European Union's Horizon 2020 research and innovation programme (666881), SVDs@ target (667375), CoSTREAM, SyNergy (EXC 2145 SyNergy - ID 390857198), the CRC 1123 (B3 and project DI 722/13-1), the Corona Foundation, the LMUexcellent fond, the e:Med programme (e:AtheroSysMed) and the FP7/2007-2103 European Union project CVgenes@ target (Health-F2-2013-601456). DG is supported by the British Heart Foundation Centre of Research Excellence (RE/18/4/34215) at Imperial College London. This work was supported by funding from the National Institute for Health Research (NIHR; Cambridge Biomedical Research Centre at the Cambridge University Hospitals NHS Foundation Trust). The views expressed are those of the authors and not necessarily those of the NHS, the NIHR or the Department of Health and Social Care.

Authors' relationships and activities DG is employed part-time by Novo Nordisk. The remaining authors have no conflicts of interest to declare.

Contribution statement DG and SB designed the study. SB, AMM and DG performed analysis. DG and SB drafted the manuscript. All authors 
interpreted the results and critically revised the manuscript for intellectual content. DG takes responsibility for the contents of the article. All authors approved the final version of this manuscript to be published.

Open Access This article is licensed under a Creative Commons Attribution 4.0 International License, which permits use, sharing, adaptation, distribution and reproduction in any medium or format, as long as you give appropriate credit to the original author(s) and the source, provide a link to the Creative Commons licence, and indicate if changes were made. The images or other third party material in this article are included in the article's Creative Commons licence, unless indicated otherwise in a credit line to the material. If material is not included in the article's Creative Commons licence and your intended use is not permitted by statutory regulation or exceeds the permitted use, you will need to obtain permission directly from the copyright holder. To view a copy of this licence, visit http://creativecommons.org/licenses/by/4.0/.

\section{References}

1. Roth GA, Johnson C, Abajobir A et al (2017) Global, regional, and national burden of cardiovascular diseases for 10 causes, 1990 to 2015. J Am Coll Cardiol 70(1):1-25. https://doi.org/10.1016/j.jacc. 2017.04.052

2. Levitan EB, Song Y, Ford ES et al (2004) Is nondiabetic hyperglycemia a risk factor for cardiovascular disease? A meta-analysis of prospective studies. Arch Intern Med 164(19):2147-2155. https:// doi.org/10.1001/archinte.164.19.2147

3. Vujkovic M, Keaton JM, Lynch JA et al (2020) Discovery of 318 new risk loci for type 2 diabetes and related vascular outcomes among 1.4 million participants in a multi-ancestry meta-analysis. Nat Genet 52(7):680-691. https://doi.org/10.1038/s41588-0200637-y

4. Wheeler E, Leong A, Liu CT et al (2017) Impact of common genetic determinants of hemoglobin A1c on type 2 diabetes risk and diagnosis in ancestrally diverse populations: a transethnic genome-wide meta-analysis. PLoS Med 14(9):e1002383. https:// doi.org/10.1371/journal.pmed.1002383

5. Burgess S, Dudbridge F, Thompson SG (2016) Combining information on multiple instrumental variables in Mendelian randomization: comparison of allele score and summarized data methods. Stat Med 35(11):1880-1906. https://doi.org/10.1002/sim.6835

6. Burgess S, Davies NM, Thompson SG et al (2014) Instrumental variable analysis with a nonlinear exposure-outcome relationship. Epidemiology 25(6):877-885. https://doi.org/10.1097/EDE. 0000000000000161

7. Cai X, Zhang Y, Li M et al (2020) Association between prediabetes and risk of all cause mortality and cardiovascular disease: updated meta-analysis. BMJ 370:m2297. https://doi.org/10.1136/bmj. m2297

8. Ahmad OS, Morris JA, Mujammami M et al (2015) A Mendelian randomization study of the effect of type- 2 diabetes on coronary heart disease. Nat Commun 6:7060. https://doi.org/10.1038/ ncomms 8060

9. Action to Control Cardiovascular Risk in Diabetes Study Group, Gerstein HC, Miller ME et al (2008) Effects of intensive glucose lowering in type 2 diabetes. N Engl J Med 358(24):2545-2559. https://doi.org/10.1056/NEJMoa0802743

10. Advance Collaborative Group, Patel A, MacMahon S et al (2008) Intensive blood glucose control and vascular outcomes in patients with type 2 diabetes. N Engl J Med 358(24):2560-2572. https://doi. org/10.1056/NEJMoa0802987

11. Kristensen SL, Rorth R, Jhund PS et al (2019) Cardiovascular, mortality, and kidney outcomes with GLP-1 receptor agonists in patients with type 2 diabetes: a systematic review and meta-analysis of cardiovascular outcome trials. Lancet Diabetes Endocrinol 7(10): 776-785. https://doi.org/10.1016/S2213-8587(19)30249-9

Publisher's note Springer Nature remains neutral with regard to jurisdictional claims in published maps and institutional affiliations. 